\title{
The relativistic iron line profile in the Seyfert 1 Galaxy IC4329a
}

\author{
C. Done ${ }^{1}$, G.M. Madejski², P.T. Życki ${ }^{3}$ \\ ${ }^{1}$ Department of Physics, University of Durham, South Road, Durham DH1 3LE, England; \\ chris.done@durham.ac.uk \\ 2 NASA/Goddard Space Flight Center, Greenbelt, MD 20771, USA; \\ madejski@lheavx.gsfc.nasa.gov \\ 3 Nicolaus Copernicus Astronomical Center, Bartycka 18, 00-716 Warsaw, Poland; \\ ptz@camk.edu.pl
}

\begin{abstract}
We present simultaneous ASCA and RXTE data on the bright Seyfert 1 galaxy IC4329a. The iron line is significantly broadened, but not to the extent expected from an accretion disk which extends down to the last stable orbit around a black hole. We marginally detect a narrow line component, presumably from the molecular torus, but even including this gives a line profile from the accretion disk which is significantly narrower that that seen in MCG-6-30-15, and is much more like that seen from the low/hard state galactic black hole candidates. This is consistent with the inner disk being truncated before the last stable orbit, forming a hot flow at small radii as in the ADAF models. However, we cannot rule out the presence of an inner disk which does not contribute to the reflected spectrum, either because of extreme ionisation suppressing the characteristic atomic features of the reflected spectrum or because the $\mathrm{X}$-ray source is intrinsically anisotropic, so it does not illuminate the inner disk.

The source was monitored by RXTE every 2 days for 2 months, and these snapshot spectra show that there is intrinsic spectral variability. The data are good enough to disentangle the power law from the reflected continuum and we see that the power law softens as the source brightens. The lack of a corresponding increase in the observed reflected spectrum implies that either the changes in disk inner radial extent/ionisation structure are small, or that the variability is actually driven by changes in the seed photons which are decoupled from the hard X-ray mechanism.
\end{abstract}

Subject headings: accretion, accretion disks - black hole physics - galaxies: individual (IC4329a) - galaxies: Seyfert - X-rays: galaxies

\section{INTRODUCTION}

Accretion of material onto a black hole is known to produce hard $\mathrm{X}$-ray ( $E \geq 2 \mathrm{keV}$ ) emission: satellite observations over the last 30 years have conclusively shown this to be the case for both 
stellar mass black holes in our own Galaxy, and the supermassive black holes which power the Active Galactic Nuclei (AGN). However, it is still the case that the mechanism by which the gravitational potential energy is converted into high energy radiation is not understood. Standard models of an accretion disk (Shakura \& Sunyaev 1973, hereafter SS) produce copious UV and even soft X-ray radiation, but are completely unable to explain the observed higher energy X-ray emission which extends from the disk spectrum out to $200 \mathrm{keV}$. Clearly something other than the standard model is required, and the two currently favored candidates are either magnetic reconnection above an accretion disk (e.g. Galeev, Rosner \& Vaiana 1979; Haardt, Maraschi \& Ghisellini 1994; di Matteo 1998), or that some part of the accretion flow is not given by the standard disk configuration.

The recent rediscovery of another stable solution of the accretion flow equations lent plausibility to this second possibility. The standard SS accretion disk model derives the accretion flow structure in the limit where the gravitational energy released is radiated locally in an optically thick, geometrically thin disk. This contrasts with the new accretion models, where below some critical mass accretion rate, $\dot{m} \leq \dot{m}_{\text {crit }}$, the material is not dense enough to thermalise and locally radiate all the gravitational potential energy which is released. Instead the energy can be carried along with the flow (advected), eventually disappearing into the black hole. These solutions give an X-ray hot, optically thin, quasi-spherical flow (Narayan \& Yi 1995; Esin, McClintock \& Narayan 1997).

Clearly it would be nice to know which (if any!) of these models for the hard X-ray emission is correct. In its most general form the problem comes down to understanding the geometry of the cool, optically thick accretion flow. If this extends down to the last stable orbit around the black hole then it is unlikely that the advective flow can exist (e.g. Janiuk, Życki \& Czerny 1999). Conversely, if the optically thick disk truncates before this point then a composite model with an outer disk and inner, hot, advective flow (ADAF) may be favored.

The accretion flow can be tracked via X-ray spectroscopy. Wherever hard X-rays illuminate optically thick material then this gives rise to a Compton reflected continuum and associated iron K $\alpha$ fluorescence line (e.g. Lightman \& White 1988; George \& Fabian 1991; Matt, Perola \& Piro 1991). The amplitude of the line and reflected continuum is dependent on the solid angle subtended by the disk to the $\mathrm{X}$-ray source (as well as on elemental abundances, inclination and ionisation). The two models outlined above can then be distinguished by the amount of reflection and line, since an untruncated disk should subtend a rather larger solid angle than a truncated one.

A survey of AGN showed that Seyfert 1 spectra are consistent with a power law X-ray spectrum illuminating an optically thick, (nearly) neutral disk, which subtends a solid angle of $\sim 2 \pi$ (Pounds et al. 1990; Nandra \& Pounds 1994). This then seems to favor the magnetic reconnection picture. However, this contrasts with the situation in the Galactic Black Hole Candidates (GBHC). These are also thought to be powered by accretion via a disk onto a black 
hole, and, in their low/hard state, show broad band spectra which are rather similar to those from AGN, but have an apparently rather smaller amount of reflection (e.g. Gierliński et al. 1997; Done \& Życki 1999). One potential drawback of this comparison is that AGN inhabit a more complex environment than the GBHC. Unification schemes for Seyfert galaxies propose that there is a molecular torus which enshrouds the nucleus. The molecular torus can also contribute to the reflected spectrum, perhaps distorting our view of the very innermost regions in AGN.

These two potential sites for the reflected component can be distinguished spectrally: any features from the accretion disk should be strongly smeared by the combination of special and general relativistic effects expected from the high orbital velocities in the vicinity of a black hole (Fabian et al. 1989), whereas the molecular torus is at much larger distances so its reflected features should be narrow. The seminal ASCA observation of the AGN MCG-6-30-15 showed that the line is so broad that it requires that the accretion disk extends down to at least the last stable orbit in a Schwarzschild metric, with no narrow component from the molecular torus (Tanaka et al. 1995; Iwasawa et al. 1996), and that the relativistically smeared material subtended a solid angle of $\sim 2 \pi$ with respect to the $\mathrm{X}$-ray source. This very clear cut result then seems to rule out the advective flows, at least in their simplest form.

However, the GBHC again show a rather different picture: they have a line which is detectably broad, but not so broad as might be expected for a disk extending all the way down to 3 Schwarzschild radii $\left(R_{\text {Schw }}=2 G M / c^{2}\right.$; Życki, Done, \& Smith 1997, 1998, 1999; Done \& Życki 1999). This is consistent with the truncated disk geometry, and so perhaps with the advective flow models. Only in the soft/high state do the GBHC seem to show the extreme relativistic smearing and large amount of reflection seen in the MCG-6-30-15 spectra (Życki et al. 1998, Gierliński et al. 1999).

Is there a real difference in accretion geometry between GBHC and AGN, pointing to a difference in radiation mechanism ? This seems unlikely, since both classes are ultimately accreting black holes. Are subtle ionisation effects masking the derived disk parameters in the GBHC (Ross, Fabian \& Young 1999). Or is MCG-6-30-15 unusual among AGN (and GBHC) in having such a relativistic disk? Perhaps MCG-6-30-15 is an AGN in a state which corresponds to the soft state GBHC?

One factor supporting the latter is a recent study by Zdziarski, Lubiński, \& Smith (1999) which showed that there is a correlation between the intrinsic spectral slope, $\Gamma$, and the solid angle subtended by the reflecting material, $\Omega / 2 \pi$. In their plots, MCG-6-30-15 is the AGN with the steepest spectrum, and highest amount of reflection. This correlation also holds for individual objects (such as NGC 5548 for AGN and Nova Muscae for the GBHC), where the intrinsic spectrum hardens as the amount of reflection decreases. This suggests that there is a universal physical mechanism and/or geometry for both classes, with perhaps a single parameter determining the state of a given source through a feedback between the geometry and physical conditions in the $\mathrm{X}$-ray emitting region. Such a feedback could be provided by e.g. soft photons 
from the thermalized fraction of the hard X-rays intercepted by the reprocessing medium, and the parameter could be the inner disk radius. Perhaps for MCG-6-30-15 (and other soft AGN and the soft state GBHC) the cool accretion disk extends down to the innermost stable orbit around the black hole with the $\mathrm{X}$-rays being powered by magnetic reconnection above the disk, while for harder spectra AGN (and the low state GBHC) the inner disk recedes outwards, being replaced by an X-ray hot (advective ?) flow. As the disk recedes it subtends a smaller solid angle, so there is less reflection (and less relativistic smearing), but there are also fewer seed photons from the disk (both from intrinsic emission and by reprocessing) for Compton scattering into the intrinsic power law, giving a harder intrinsic power law (Życki et al. 1999; Zdziarski et al. 1999).

If this is true, then this clearly predicts that the $\mathrm{Fe} \mathrm{K} \alpha$ line in $A G N$ is not always as broad as in the extreme case of MCG-6-30-15 (Tanaka et al. 1995). Previous ASCA studies on a sample of objects (Nandra et al. 1997) hint towards such a possibility, since a whole range of geometries were inferred for various objects.

Here we test this idea using ASCA, XTE and OSSE data from IC 4329a, the brightest 'typical' Seyfert 1 in the X-ray band (cf. Madejski et al. 1995). It lies towards the middle of the $\Gamma-\Omega / 2 \pi$ plot of Zdziarski et al. (1999), and (consequently) has a spectrum very close to that of the mean Seyfert 1 spectrum compiled by Zdziarski et al. (1995). IC 4329a may then be used as a template for Seyfert galaxies as a class, unlike MCG-6-30-15 which has a rather steep X-ray spectral index.

\section{DATA REDUCTION}

\subsection{ASCA}

The 1997 campaign for IC 4329a included four ASCA pointings, on August 7, 10, 12, and 16, each nominally providing $20 \mathrm{ks}$ of data. The resulting data were extracted using the standard screening procedures, yielding total exposures of $61 \mathrm{ks}$ for SIS0 and SIS1 (using the BRIGHT2 mode), and $78 \mathrm{ks}$ for GIS2 and GIS3. The source data were extracted from circular regions with radii of 3 arc min for the SISs and 4 arc min for the GISs, while background was taken from a source-free regions of the same images. The source showed a clear variability between the four pointings, with GIS2 counting rates of $1.77 \pm 0.009,1.27 \pm 0.008,1.53 \pm 0.009$, and $1.78 \pm 0.009$, matching the variability seen in the simultaneous RXTE data (see below and Fig. 1). The PHA data were then grouped so as to have more than 20 counts per bin.

\subsection{RXTE: PCA}

IC4329a was observed a total of 66 times over a period of 58 days with RXTE. The PCA standard 2 data were extracted from all layers of detectors 0,1 and 2 , using standard selection 
criteria (Earth elevation angle $>10^{\circ}$, offset between the source and the satellite pointing direction $<0.02^{\circ}$, electron rates in each detector $<0.1$, excluding data taken within 30 minutes of the SAA passage). This gave a total of $73 \mathrm{ks}$ of good data. The background for these data were then modeled using the 'L7' model (see Zhang et al. 1993; Madejski et al. 1999; and Jahoda et al. 2000, in preparation), and the corresponding response matrix for each observation was generated using version 3.0 of the channel to energy conversion file. We use data from $3-20 \mathrm{keV}$, since lower energies are affected by response matrix uncertainties and the background may not be well modeled above $20 \mathrm{keV}$. A $1 \%$ systematic error is applied to data in all PHA channels.

\subsection{RXTE: HEXTE}

The HEXTE instrument onboard RXTE (cf. Rothschild et al. 1998) consists of two scintillator modules, sensitive in the hard X-ray band. The background is measured via chopping the detectors to off-axis, source free locations every 16 seconds. The HEXTE data were extracted using standard data reduction procedures, which, after appropriate dead-time correction, yielded a total net exposure of $\sim 24 \mathrm{ks}$. The source was clearly detected in each pointing over the range of $15-100 \mathrm{keV}$ range, at a level consistent with the PCA, but the statistical errors in each pointing were too large to study variability. The resultant data were binned such that channels 17-28 (16.9-28.3 keV) were grouped by a factor $3,29-48(28.3-47.8 \mathrm{keV})$ by a factor 4 and $49-120$ (47.8-123.4 keV) by a factor 6 . The effective area of both HEXTE clusters was scaled by 0.7 , the current best normalization to the Crab spectrum.

\subsection{OSSE}

OSSE pointed at IC 4329a during the CGRO viewing period 625, over the epoch of 1997 August 8 to 18, with the total exposure of $567 \mathrm{ks}$. The data were reduced in a standard manner (see Johnson et al. 1997 and references therein), resulting in a net counting rate of $0.18 \pm 0.08$ counts $\mathrm{s}^{-1}$ over the $50-500 \mathrm{keV}$ range. The resulting data were binned such that channels 9-18 were grouped by a factor 5 , while 19-48 were grouped by a factor 10 .

\subsection{Lightcurves}

Figure 1 shows the $2-10 \mathrm{keV}$ light curve obtained from the RXTE PCA data. The source is clearly variable on timescales of a few days, with an r.m.s variation of $13 \%$. There is no significant short timescale variability within each observations. These typically have $3 \sigma$ upper limits of 0.06 to the fractional r.m.s variability of the 1000-2000 second lightcurve binned on 16 seconds. The horizontal lines on Figure 1 mark the times at which ASCA and OSSE data were taken. 


\section{SPECTRAL FITTING}

The data were fitted using XSPEC v10.0, with errors quoted as $90 \%$ confidence intervals $\left(\Delta \chi^{2}=2.7\right)$. We use elemental abundances and cross-sections of Morrison \& McCammon (1983).

\subsection{ASCA SIS and GIS}

Data from SIS0 and SIS1 are showing increasing divergence with time from the GIS2 and GIS3 (and from each other) at low energies. The reasons for this are not yet well understood (Weaver \& Gelbord 1999, in preparation). A current pragmatic approach is to allow excess absorption in the SIS detectors to account for this effect to zeroth order.

Another source of low energy complexity is the partially ionized absorber, first seen in the ROSAT PSPC spectrum (Madejski et al. 1995). A previous ASCA observation has shown that in IC4329a this complex absorption is better modeled by two edges (corresponding to $\mathrm{H}$ and He-like Oxygen at rest energies of 0.739 and $0.871 \mathrm{keV}$, respectively) rather than a full ionized absorber code (Cappi et al. 1996; Reynolds 1997 see also the discussion in George et al. 1998). We use this description here, but we caution that the determination of this absorption in our data will be somewhat dependent on the way the low energy calibration problems are treated.

We first use a phenomenological model for the ASCA data, consisting of an underlying power law and its reflected continuum from a neutral disk (pexrav: Magdziarz \& Zdziarski 1995) inclined at $30^{\circ}$, together with a separate Gaussian iron line, with the two edge description for the warm absorber. This model gives a good fit to the data $\left(\chi_{\nu}^{2}=2533 / 2392\right)$, for an intrinsic power law spectrum of $\Gamma=1.85 \pm 0.03$, and reflector solid angle (for an inclination of $30^{\circ}$ ) of $\Omega / 2 \pi=0.48_{-0.31}^{+0.34}$. The associated iron $\mathrm{K} \alpha$ fluorescence line is at a (rest frame) energy of $6.37 \pm 0.06 \mathrm{keV}$, with equivalent width of $180 \pm 50 \mathrm{eV}$ and intrinsic width of $\sigma=0.39 \pm 0.10 \mathrm{keV}$ (hereafter all intrinsic line widths are the gaussian $\sigma$ ).

The iron line physical width is similar to that seen in a previous observation of this AGN (Mushotzky et al. 1995; Cappi et al. 1996; Reynolds 1997), but is much smaller than that seen from the archetypal relativistically smeared line in MCG-6-30-15 (Tanaka et al. 1995). One way to show this is to fit the spectrum in the $2.5-10 \mathrm{keV}$ band (where the effects of the ionised absorption is much less) with a simple power law and its expected reflected continuum (with solar abundances, and fixed solid angle $\Omega / 2 \pi=1$ at $30^{\circ}$ inclination). The fit excludes the $5-7 \mathrm{keV}$ iron line range. Figure 2a shows the resulting shape of the line residuals from IC4329a, while figure $2 \mathrm{~b}$ shows those from MCG-6-30-15 for comparison.

Replacing the Gaussian line with a diskline relativistic model (fixing the disk inclination at $30^{\circ}$ and line emissivity at $\alpha r^{-\beta}$ with $\beta=3$ ) gives that the smearing implies an inner disk radius of $48_{-20}^{+33} R_{\mathrm{g}}\left(\chi_{\nu}^{2}=2540 / 2392\right.$ : a slightly worse fit than the simple broad Gaussian line). This is significantly larger than the last stable orbit at $6 R_{g}\left(R_{\mathrm{g}}=G M / c^{2}\right.$, so the last stable orbit at 3 


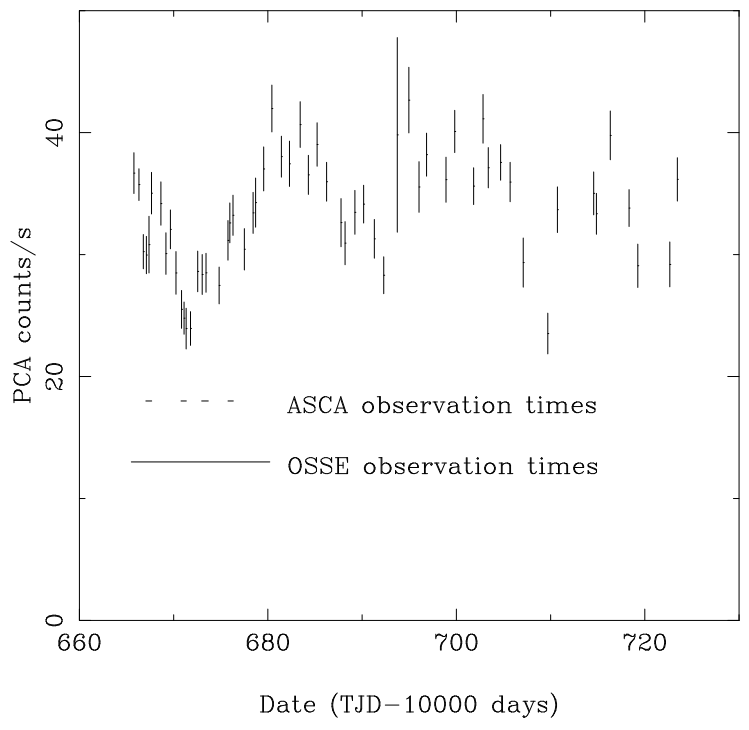

Fig. 1. - XTE PCA count rate showing the variability throughout the monitoring campaign. The times of the ASCA and OSSE observations are marked by the horizontal lines
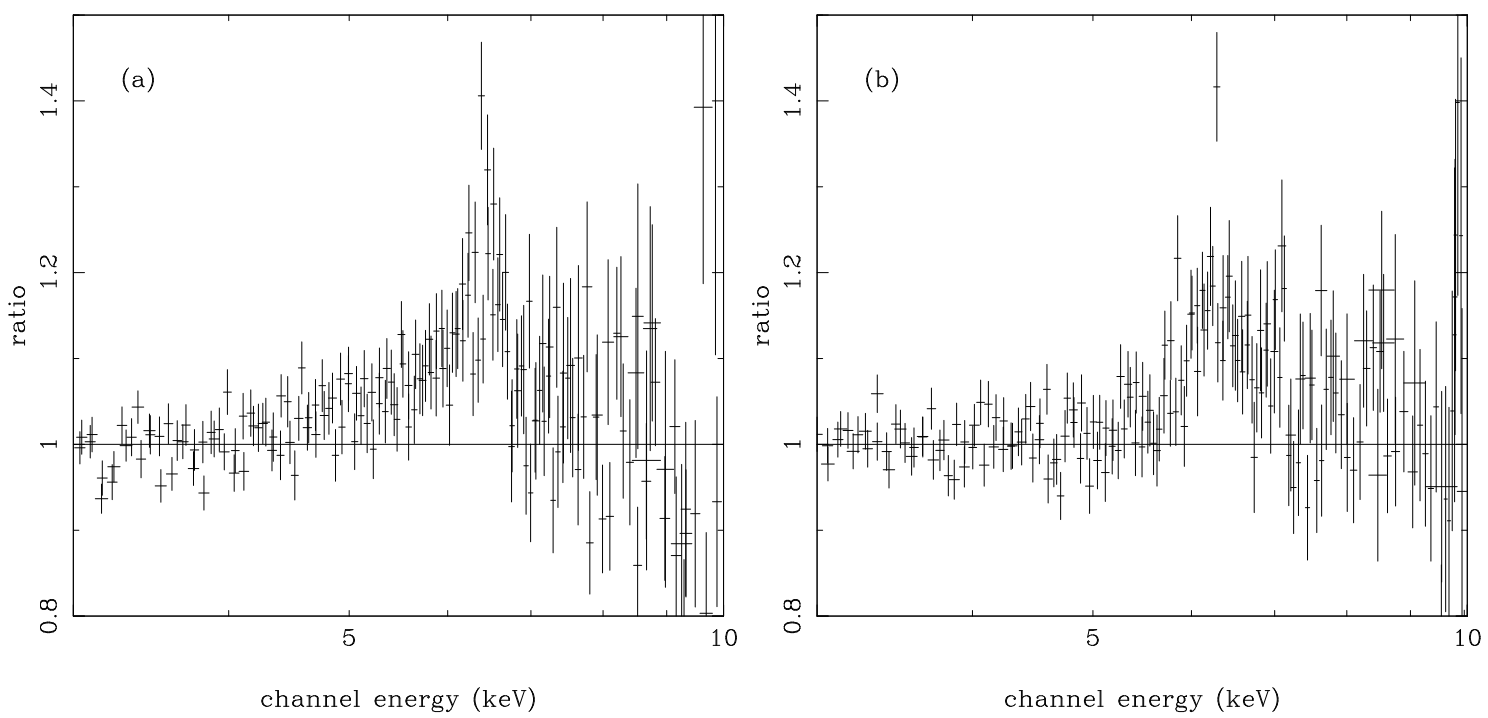

Fig. 2.- Residuals of a continuum fit to a power law plus its Compton reflected component over the 2.5-10 keV bandpass, excluding the iron line range of 5-7 keV. (a) shows the residuals to the ASCA IC4329a data presented here, while (b) shows residuals to the ASCA long look observation of MCG-6-30-15 of Tanaka et al (1995). The line is clearly broader and skewed towards lower energies in MCG-6-30-15 than in IC4329a. 
Schwarzchild radii is at $6 R_{\mathrm{g}}$ ). The derived radius is dependent on the form of the emissivity, but fits to two separate observations of MCG-6-30-15 require $\beta=3.4_{-0.8}^{+1.3}$ and $\beta=4.4_{-1.1}^{+3.0}$, respectively (Nandra et al 1997), showing that the line emission is strongly weighted towards the innermost radii. We might also expect this on theoretical arguments. The energy emitted per unit area of a disk goes as $r^{-3}$, so this should give the time averaged emissivity from a magnetic corona, as well as being a good approximation to the illumination expected from a central spherical source (see appendix A in Życki et al 1999). Thus we fix $\beta=3$ in all our fits, which gives $\Delta \chi^{2}>20$ for an inner radius equal to the last stable orbit at any inclination.

The reflection description used above has several drawbacks. Firstly it allows the line to vary in intensity (and energy) without reference to the reflected continuum. Secondly, the relativistic smearing is only applied to the iron line, and not to the reflected continuum also. We replace these components with the reflection model described by Życki et al. (1999), rel-repr, which calculates the self-consistent line associated with the reflected continuum, and then applies the relativistic smearing (including gravitational light bending) to this total reprocessed spectrum. The ionisation state is a free parameter, and the models are calculated for iron abundance between $1-2 \times$ solar. The reprocessor in AGN is generally assumed to be neutral but an accretion disk temperature of $\sim 10 \mathrm{eV}$ can give a thermal population dominated by ions with ionization potential $\sim 10-30 \times k T$ (Rybicki \& Lightman 1979) i.e. between Fe V - FeX, which in our model corresponds to $0.3<\xi<20$. Photo-ionisation by the $\mathrm{X}$-ray source can increase these estimates considerably.

We replace the pexrav and diskline components with our combined model for the reprocessed component. We allow the ionisation, iron abundance, and inner disk radius to be fit

parameters, for a fixed inclination of $30^{\circ}$, and for a fixed line emissivity of $\propto r^{-3}$. This again gave a disk inner radius of $45_{-18}^{+32} R_{\mathrm{g}}\left(\chi_{\nu}^{2}=2540 / 2392\right)$.

\subsection{ASCA-XTE Simultaneous Data}

We extracted RXTE data which were taken exactly simultaneously with the ASCA observation (datasets 4,5,6,11,12,13,16,16-01,18,19 and 20), giving a total PCA exposure of $13 \mathrm{ks}$. The corresponding HEXTE data over this short time interval have very low signal to noise so do not add any appreciable constraints. Figure 3a shows the residuals resulting from an absorbed power law fit to the PCA data, showing the classic signature of Compton reflection and its associated iron fluorescence line $\left(\chi_{\nu}^{2}=162.3 / 42\right)$. Figure $3 \mathrm{~b}$ shows residuals including a broad gaussian line in the fit. Clearly there are still systematic residuals, with a decrement at the expected energy of the iron edge and a rise to higher energies $\left(\chi_{\nu}^{2}=50.5 / 39\right)$. Including a reflected continuum component (the pexrav model in xspec, assuming solar abundances and inclination of $30^{\circ}$ ) gives $\chi_{\nu}^{2}=11.7 / 38$, showing that the reflected continuum component is significantly detected independently of the iron line. 
Since the data are simultaneous, we can fix the absorption (warm and cold) to that seen in the GIS for the same model fit to the ASCA data. This gives an excellent fit to the data with $\chi_{\nu}^{2}=12.6 / 39$ (rather too good in fact, showing that the statistics are dominated by the 1 per cent systematic error) for an intrinsic power law spectrum of $\Gamma=1.94 \pm 0.04$, and reflector solid angle (for an inclination of $30^{\circ}$ ) of $\Omega / 2 \pi=0.49_{-0.14}^{+0.17}$. The associated iron $\mathrm{K} \alpha$ fluorescence line is at a (rest frame) energy of $6.33 \pm 0.13 \mathrm{keV}$, with equivalent width of $210 \pm 45 \mathrm{eV}$ and intrinsic width (gaussian $\sigma$ ) of $0.49_{-0.18}^{+0.19} \mathrm{keV}$. The PCA 2-10 keV flux is 25 per cent higher than that from ASCA due to absolute flux calibration problems in both instruments: ASCA gives a Crab 2-10 keV flux of $1.8 \times 10^{-8} \mathrm{ergs} \mathrm{s}^{-1}$ (Makishima et al., 1996), while RXTE gives $2.4 \times 10^{-8} \mathrm{ergs} \mathrm{s}^{-1}$ (see PCA Crab spectrum at http://lheawww.gsfc.nasa.gov/users/keith/pcarmf.html) and the original Crab calibration is $\sim 2.15 \times 10^{-8} \mathrm{ergs} \mathrm{s}^{-1}$ (Toor \& Seward 1974). A more serious discrepancy is in the spectral index, which is $\Delta \alpha \sim 0.1$ steeper than that seen in ASCA. It is known that the current RXTE PCA calibration gives results for the Crab which are roughly $\Delta \alpha \sim 0.1$ steeper than the index assumed for the calibration of other instruments (K. Jahoda, private communication). However, reassuringly, the relative amount of reflection equivalent width, energy and intrinsic width of the line are consistent with those from the ASCA data, though of course the absolute normalisations are different due to the calibration discrepancies. Tieing the absorption across the two datasets, together with the relative reflection parameters (the reflector solid angle, its inner radius and ionisation state, and the iron line energy, width and equivalent width) gives $\chi_{\nu}^{2}=2548 / 2435$, not significantly different from the $\chi_{\nu}^{2}=2546 / 2432$ obtained from the separate fits. Thus in what follows we tie the relative reflection (and absorber) parameters across the two datasets, and let only the power law spectral index and normalization be free.

We replace the pexrav and broad Gaussian line with our rel-repr model. Table 1 shows the results of a joint fit to the ASCA and RXTE PCA data for inclination angles of 30,60 and $72^{\circ}$ and for iron abundances of 1 and $2 \times$ solar. The derived inner disk radius is highly correlated with the assumed inclination. High inclination angles give stronger Doppler effects and so a broader line. The inner disk radius then has to be larger to match the observed line width. However, none of the fits allow the disk to extend down to the innermost stable orbit, irrespective of inclination. This also means that the data are not very sensitive to the inclination $\left(\Delta \chi^{2} \sim 4\right.$, i.e. marginally significant preference for higher inclinations). It is only the broadest components from the very innermost disk which significantly change the skewness of the line profile (as opposed to its width) as function of inclination.

The data are sensitive to the iron abundance. They significantly prefer supersolar abundances $\left(\Delta \chi^{2} \geq 7\right)$, as would be expected from measurements of radial abundance gradients in spiral galaxies (e.g. Henry \& Worthey 1999). Another way to see this is the phenomenological fits give a line equivalent with of $180 \mathrm{eV}$, as expected for a solar abundance slab subtending a solid angle of $2 \pi$ (e.g. George \& Fabian 1991), yet the solid angle of the reflected continuum in these fits is approximately half of this. Figure 4 shows the best fit joint RXTE and ASCA data fit to a disk model with $2 \times$ solar abundance, inclined at $30^{\circ}$. 

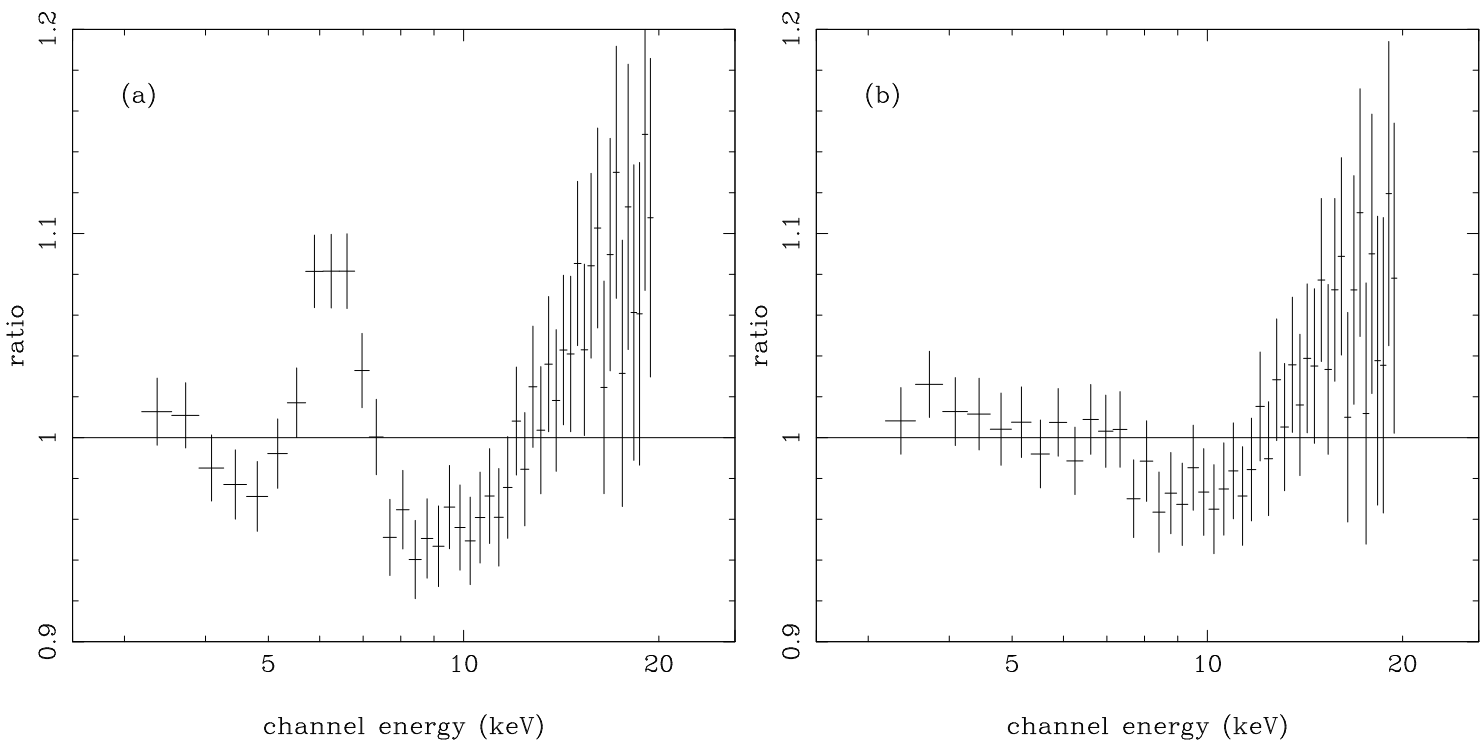

Fig. 3.- XTE data: (a) shows residuals to a simple power law, showing the classic reflection signature. (b) shows the remaining residuals including a broad gaussian line. Plainly the reflected continuum is detected independently of the iron line.

Table 1. ASCA and simultaneous XTE PCA data fit to the rel-repr model

\begin{tabular}{|c|c|c|c|c|c|c|c|}
\hline $\mathrm{A}_{\mathrm{Fe}}{ }^{\mathrm{a}}$ & Inclination & $\begin{array}{c}\Gamma^{\mathrm{b}} \\
\mathrm{ASCA}, \mathrm{XTE}\end{array}$ & $\begin{array}{c}\text { Norm at } 1 \mathrm{keV} \\
\text { ASCA, XTE }\end{array}$ & $\Omega / 2 \pi^{\mathrm{c}}$ & $\xi^{\mathrm{d}}$ & $R_{\text {in }}{ }^{\mathrm{e}}\left(R_{\mathrm{g}}\right)$ & $\chi_{\nu}^{2}$ \\
\hline 1 & 30 & $1.87 \pm 0.02,1.99_{-0.04}^{+0.03}$ & $0.031,0.049$ & $0.62_{-0.13}^{+0.11}$ & $21_{-13}^{+45}$ & $35_{-15}^{+20}$ & $2567 / 2436$ \\
\hline 1 & 60 & $1.88 \pm 0.02,2.00_{-0.02}^{+0.04}$ & $0.031,0.05$ & $0.85_{-0.10}^{+0.16}$ & $12_{-8}^{+10}$ & $90_{-30}^{+60}$ & $2575 / 2436$ \\
\hline 1 & 72 & $1.89 \pm 0.02,2.02 \pm 0.04$ & $0.031,0.051$ & $1.25_{-0.20}^{+0.23}$ & $7_{-5}^{+10}$ & $110_{-38}^{+80}$ & $2576 / 2436$ \\
\hline 2 & 30 & $1.85 \pm 0.02,1.94_{-0.02}^{+0.03}$ & $0.030,0.046$ & $0.55 \pm 0.10$ & $4_{-3.95}^{+11}$ & $35_{-12}^{+16}$ & $2562 / 2436$ \\
\hline 2 & 60 & $1.86_{-0.02}^{+0.01}, 1.97 \pm 0.02$ & $0.030,0.048$ & $0.88 \pm 0.11$ & $0.01_{-0.01}^{+0.8}$ & $75_{-23}^{+45}$ & $2560 / 2436$ \\
\hline 2 & 72 & $1.86 \pm 0.02,1.95_{-0.02}^{+0.03}$ & $0.030,0.048$ & $1.20_{-0.10}^{+0.13}$ & $0.005_{-0.005}^{+0.3}$ & $90_{-25}^{+40}$ & $2558 / 2436$ \\
\hline
\end{tabular}

${ }^{\text {a } I r o n ~ a b u n d a n c e ~ o f ~ r e f l e c t o r ~ r e l a t i v e ~ t o ~ s o l a r ~}$

${ }^{\mathrm{b}}$ Photon spectral index

${ }^{\mathrm{c}}$ Solid angle subtended by the reprocessor with respect to the $\mathrm{X}$-ray source

${ }^{\mathrm{d}}$ Ionization parameter of the reprocessor

${ }^{\mathrm{e}}$ Inner radius of the accretion disk 


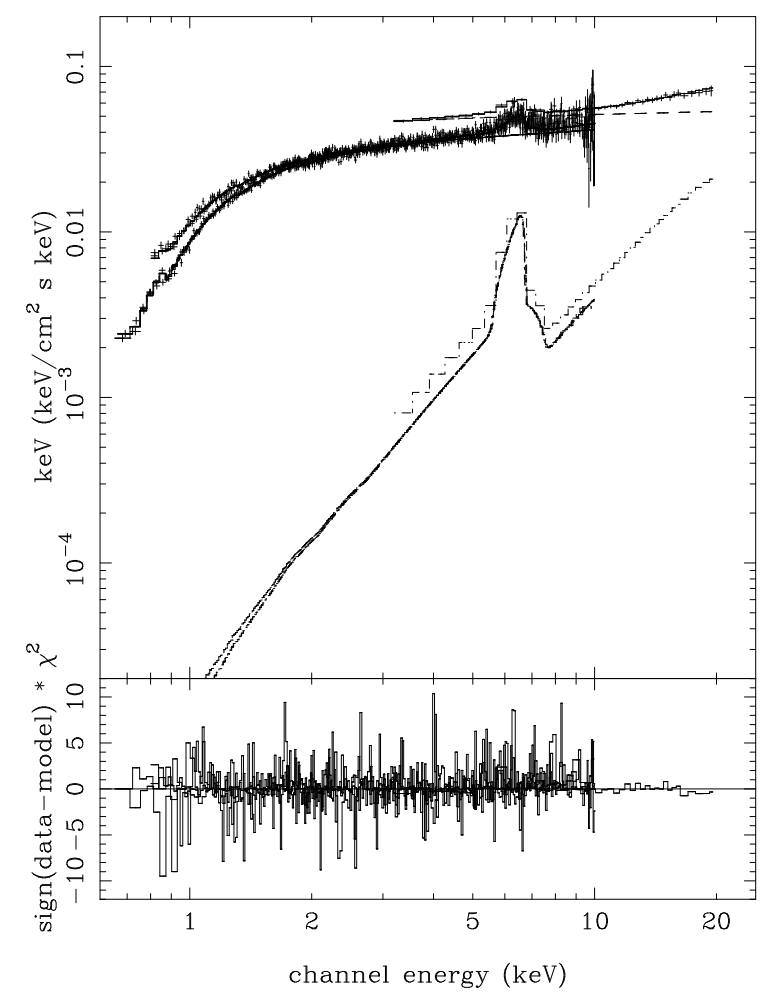

Fig. 4.- ASCA and simultaneous RXTE data fit to a model in which the relativistic reprocessor is assumed to have twice solar iron abundance, and is inclined at $30^{\circ}$. The ASCA and RXTE intrinsic power law index is not tied between the two instruments due to calibration uncertainties. 
Since IC4329a is a Seyfert 1 there could be a contribution to the line/reflected continuum from a molecular torus as well as from the accretion disk (Ghisellini, Haardt \& Matt 1994; Krolik, Madau \& Życki 1994). Table 2 shows the results obtained including a neutral, unsmeared reflector, with assumed mean inclination of $60^{\circ}$. Only the parameters of the two reflectors are included, since the continuum is similar to that derived before. This gives a significantly better fit to the data, generally with $\Delta \chi^{2} \geq 9$ for the addition of 1 extra free parameter (the amount of non-relativistic reflection).

The double reprocessing model now gives a fit which is as good as or even better than those from the phenomenological (i.e. unphysical) broad Gaussian line/pexrav model. The observed line is fairly broad but also fairly symmetric, contrary to the predictions of a line from an accretion disk which must also be skewed if it is broad. Adding the second neutral, non-smeared reprocessor gives a narrow core to the line, while the line from the relativistic reprocessor then fills in a broad (and skewed) line wing. The presence of the narrow component means that the relativistic effects have to be more marked than in the single reflector fits in order to make the total line as broad as before. Thus the derived inner disk radius is always rather smaller than before but still never consistent with the 3 Schwarzschild radii. The smaller inner radius means that the lower inclination reflected spectra are significantly gravitationally redshifted, so requiring the reflector to be somewhat ionized to compensate for this.

The above discussion assumed that the torus was Compton thick, i.e. with $N_{H} \gg 10^{24}$ $\mathrm{cm}^{-2}$. However, it can still produce substantial line emission, without the accompanying strong reflected component if the torus column is $\sim 10^{23-24} \mathrm{~cm}^{-2}$. Replacing the unsmeared, neutral reflected component by a narrow $6.4 \mathrm{keV}$ line gives a similar series of fits as those shown in Table 2. In particular, the fits never allow the amount of smearing to be as large as expected from the innermost stable orbit of an accretion disk, and they show the same preference for twice solar iron abundance and inclination angles $>30^{\circ}$.

The series of fits above show that the best physical description of the data is with two reprocessed components, one which is relativistically smeared and possibly ionized from the accretion disk, and one which is neutral and unsmeared (and possibly consisting of just line rather than line and reflected continuum) from the molecular torus. A similar composite line is seen in the Seyfert MCG-5-23-16 (Weaver et al. 1997; see also Weaver \& Reynolds 1998). The data prefer models with twice solar abundances, and inclinations of $>30^{\circ}$, and these solutions have the advantage that the derived ionisation of reflector is generally rather low, consistent with that expected (see section 2). However, there is a further constraint on the inclination, since the extra reprocessor cannot be along our line of sight to the nucleus. IC4329a is classified optically as a Seyfert 1 and the $\mathrm{X}$-ray spectrum is not heavily absorbed. For a disk inclined at $72^{\circ}$ then for our line of sight not to intercept the molecular torus severely restricts its scale height, and so the possible solid angle it can subtend. Thus while the range of double reflector fits given in Table 2 are statistically indistinguishable, these consistency arguments lead us to favor viewing angles to the accretion disk of $\sim 45^{\circ}$ and supersolar abundances. 
Table 2. ASCA and simultaneous XTE PCA data fit to the rel-repr model, with relativistic disk and neutral, unsmeared reflection

\begin{tabular}{lcccccc}
\hline \hline $\mathrm{A}_{\mathrm{Fe}}{ }^{\mathrm{a}}$ & Inclination & $\Omega / 2 \pi^{\mathrm{b}}$ & $\Omega / 2 \pi^{\mathrm{c}}$ & $\xi^{\mathrm{d}}$ & $R_{\text {in }}{ }^{\mathrm{e}}\left(R_{\mathrm{g}}\right)$ & $\chi_{\nu}^{2}$ \\
\hline 1 & 30 & $0.40 \pm 0.14$ & $0.25_{-0.07}^{+0.09}$ & $120_{-50}^{+110}$ & $18_{-6}^{+10}$ & $2550 / 2435$ \\
1 & 60 & $0.52_{-0.14}^{+0.13}$ & $0.33_{-0.14}^{+0.09}$ & $100_{-55}^{+90}$ & $42_{-18}^{+25}$ & $2551 / 2435$ \\
1 & 72 & $0.53_{-0.14}^{+0.13}$ & $0.46_{-0.14}^{+0.24}$ & $90_{-55}^{+70}$ & $55_{-24}^{+30}$ & $2551 / 2435$ \\
2 & 30 & $0.29 \pm 0.11$ & $0.25_{-0.15}^{+0.09}$ & $60_{-50}^{+100}$ & $16_{-5}^{+10}$ & $2552 / 2435$ \\
2 & 60 & $0.33_{-0.11}^{+0.10}$ & $0.51_{-0.18}^{+0.23}$ & $5_{-5}^{+40}$ & $32_{-10}^{+21}$ & $2545 / 2435$ \\
2 & 72 & $0.31 \pm 0.12$ & $0.85_{-0.35}^{+0.21}$ & $0.06_{-0.06}^{+25}$ & $40_{-12}^{+28}$ & $2544 / 2435$ \\
& & & & & & \\
\hline
\end{tabular}

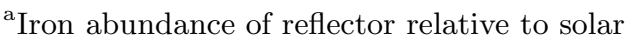

${ }^{\mathrm{b}}$ Solid angle subtended by the neutral, unsmeared reprocessor (assumed mean inclination of $60^{\circ}$ ) with respect to the $\mathrm{X}$-ray source

${ }^{\mathrm{c}}$ Solid angle subtended by the relativistic reprocessor with respect to the $\mathrm{X}$-ray source

${ }^{\mathrm{d}}$ Ionization parameter of the relativistic reprocessor

${ }^{\mathrm{e}}$ Inner radius of the relativistic accretion disk 
All these models assumed that the ionisation state of the disk was constant with radius. A more physical picture might be one where the ionisation varies as a function of radius (e.g. Matt et al. 1993). In such models, the inner disk might be so ionized that it produces no spectral features. The observed reflected spectrum would then arise from further out in the disk, and so not contain the highly smeared components. This might provide an alternative explanation to a truncated disk as to why the relativistic smearing observed is not compatible with a disk extending down to the last stable orbit. We test this by dividing the disk into 10 radial zones, with ionisation $\xi(r) \propto r^{-4}$ as described in Done \& Życki (1999). With the inner radius fixed at $6 R_{\mathrm{g}}$, and allowing for a narrow component from the molecular torus we are never able to obtain fits within $\Delta \chi^{2} \sim 20$ of those in Table 2.

\subsection{RXTE PCA Variability}

There is clear intensity variability during the RXTE campaign, and also spectral variability in the sense that the spectrum becomes softer as the source brightens. This could be due to either intrinsic change in the power law spectral index, or a change in the relative contribution of the reflected spectrum (or both).

We can attempt to disentangle the power law from the reflected spectrum by fitting these components to the individual spectra from each orbit (including a 1 per cent systematic uncertainty), fixing the absorption at $3 \times 10^{21} \mathrm{~cm}^{-2}$. The reflected spectrum is assumed to have twice solar iron abundance, be inclined at $60^{\circ}$ and have fixed negligible ionisation $(\xi=0.01$, see table 1). Thus the free parameters are the power law index and normalization, the solid angle subtended by the relativistic reflector and its inner radius. Figure 5a shows these derived parameters as a function of time. Clearly the inner radius cannot be constrained, but the index and the solid angle of the reflector show some possible trend. Fitting these with a constant gives $\chi_{\nu}^{2}=36.0 / 58$ and $13.4 / 58$ respectively i.e. they are statistically consistent with a constant value. Figure $5 \mathrm{~b}$ shows these plotted against the $2-10 \mathrm{keV}$ flux, and a linear regression (taking errors in both $x$ and $y$ into account: Press et al. 1992) shows that the power law index is significantly correlated with flux, since it gives $\chi_{\nu}^{2}=25.7 / 57$. This corresponds to an $\mathrm{F}$ value of $10.3 /(25.7 / 57)=22.8$, significant at $\geq 99.9$ per cent. Even using just the difference in $\chi^{2}$ between the two fits gives $F=10.3$, significant at $\geq 99.5$ per cent, so the correlation is clearly present. IC4329a then becomes only the second Seyfert 1 where there is clear intrinsic spectral variability (the other is NGC 5548: Magdziarz et al. 1998), where underlying continuum changes can be unambiguously disentangled from changes in the reflected spectrum.

We use the same procedure to look for variability in the amount of reflection as a function of

flux. The linear regression gives $\chi_{\nu}^{2}=10.6 / 57$ where the fractional amount of reflection relative to the power law decreases as the flux increases. This is significant at $>99.9$ per cent confidence on an $\mathrm{F}$ test, but is only 90 per cent significant using just the change in $\chi^{2}$. 

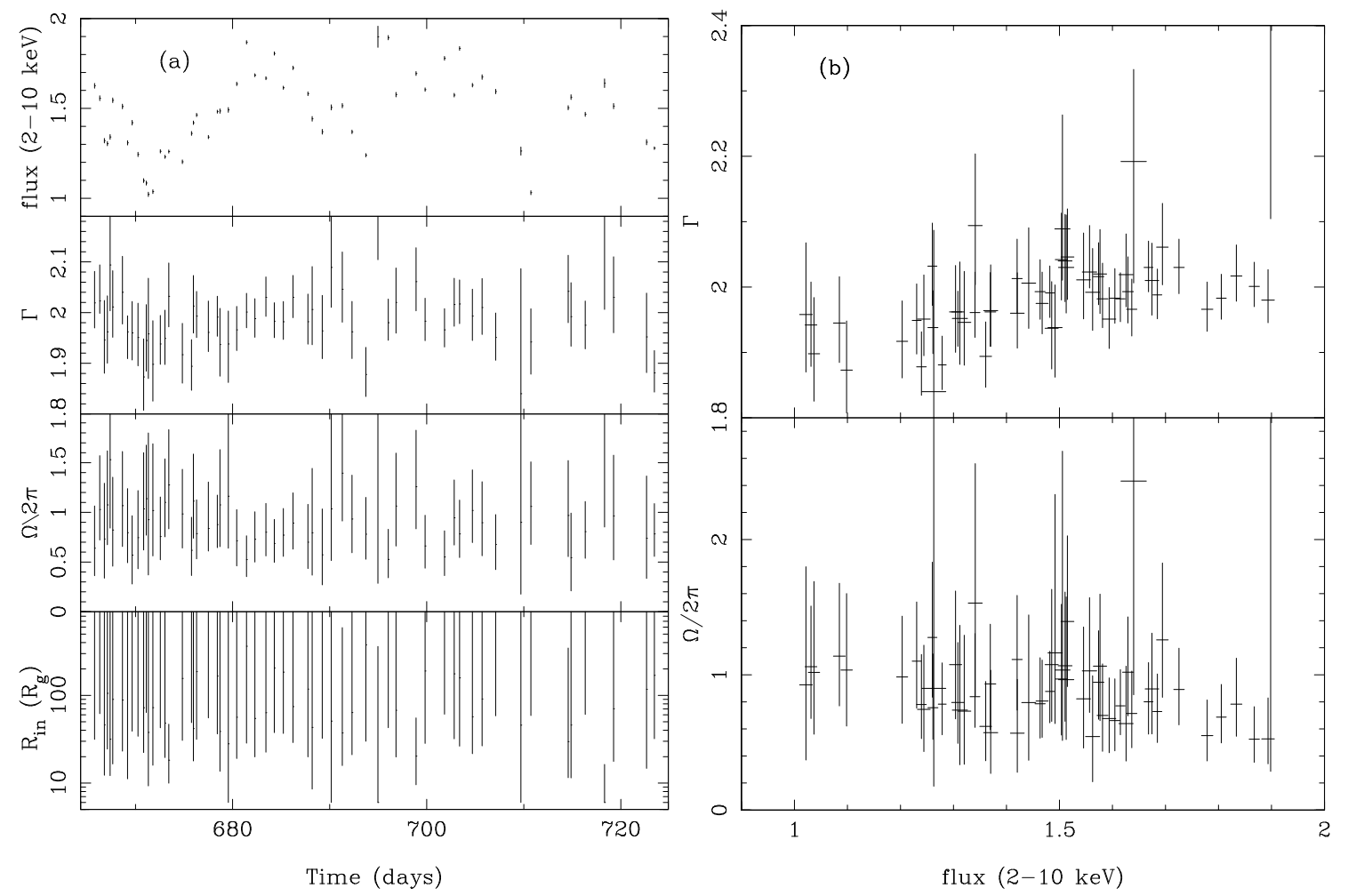

Fig. 5.- Results for fitting a simple power law and its relativistic reflection with absorption fixed at $3 \times 10^{21} \mathrm{~cm}^{-2}$ to the $3-20 \mathrm{keV}$ individual snapshot PCA spectra. (a) shows the inferred 2-10 $\mathrm{keV}$ flux, spectral index and derived inner disk radius as a function of time, while (b) shows the spectral index and amount of reflection as a function of $2-10 \mathrm{keV}$ flux. The power law becomes intrinsically steeper as the source brightens, while the reflected fraction marginally decreases. 
To illustrate these points we co-add spectra when the source was at its lowest and highest intensity level (see Figure 1), and fit these spectra with a power law and single relativistically smeared reflector (with reflector parameters fixed as above, and with the disk inner radius fixed at $60 R_{\mathrm{g}}$ ). The spectral index changes from $1.92 \pm 0.04$ to $2.00 \pm 0.02$, for the low and high state, respectively. Figure 6 shows the unfolded spectra, with the model extrapolated out to $100 \mathrm{keV}$. The thick and thin lines show the model components for the high and low state data, respectively. The underlying continuum is brighter at all energies $\leq 100 \mathrm{keV}$ in the high state despite it being steeper, with an integrated $0.01-300 \mathrm{keV}$ flux of 6.2 to $10.7 \times 10^{-10} \mathrm{ergs} \mathrm{cm}^{-2} \mathrm{~s}^{-1}$ for the low and high state, respectively. This behaviour is fairly easy to reproduce in comptonisation models in which the seed photons vary (see discussion), but the (marginally significant) lack of change in the absolute amount of reflection (so that the relative reflected fraction in the low state is larger than in the high state) is harder to explain. Clearly a model in which the reflected flux is produced at large distances from the source would be viable, but the reflected spectrum is broadened, so does contain at least some contribution from the relativistically smeared inner disk. For a $10^{8} M_{\odot}$ black hole, then the inner 100 Schwarzschild radii are on scales of $3 \times 10^{15} \mathrm{~cm}$, i.e. less than 1 lightday. The spectra are taken at intervals of a day or more, so the relativistically smeared reprocessed component should not be appreciably lagged behind the source variability.

One possibility is that this anti-correlation of relative reflected fraction with flux is intrinsic to the source, that the geometry changes in such a way as to produce less reflection as the source brightens and steepens. However, it is very hard to see how this can be the case. A brighter, steeper source implies that there are more seed photons for the Compton scattering (see Figure 6 and the Discussion), i.e. that the geometry is such that more disk photons are intercepted by the source. Thus we can easily explain more reflected flux as the source steepens, but not less. A correlation of reflected solid angle with spectral index is indeed seen in both Galactic Black Hole Candidates (e.g. Życki et al. 1999) and AGN (Zdziarski et al. 1999). It seems much more likely that the (marginal) anti-correlation is an artifact of there being a second reflector at much larger distances from the source. The light travel time delay then means that the distant reprocessor does not have time to respond to rapid flux variations, so that as the source dims the relative contribution of the reflected spectrum from the distant reprocessor increases.

We include a reprocessed component from a torus, fixing its parameters to the best fit XTE values from the joint ASCA-RXTE fit (see table 2, again assuming twice solar abundance and inclination of $60^{\circ}$, but this time also fixing the inner edge of the disk to $60 R_{\mathrm{g}}$ ). The significance of the correlation of the spectral index with flux is unchanged $\left(\chi_{\nu}^{2}=44.7 / 58\right.$ for a constant while adding the linear term gives $\left.\chi_{\nu}^{2}=29.4 / 57\right)$, while the flux/accretion disk reflection variability is now consistent with a constant solid angle $\left(\chi_{\nu}^{2}=12.4 / 58\right)$ : adding a linear term gives an insignificant change $\left(\chi_{\nu}^{2}=11.4 / 58\right)$. The results are similar for a fixed Gaussian line rather than a full reprocessed spectrum.

We illustrate this again by fits to the high and low state spectra. Figure 7a shows the confidence contours for the power law spectral index, while Figure $7 \mathrm{~b}$ shows the derived solid 


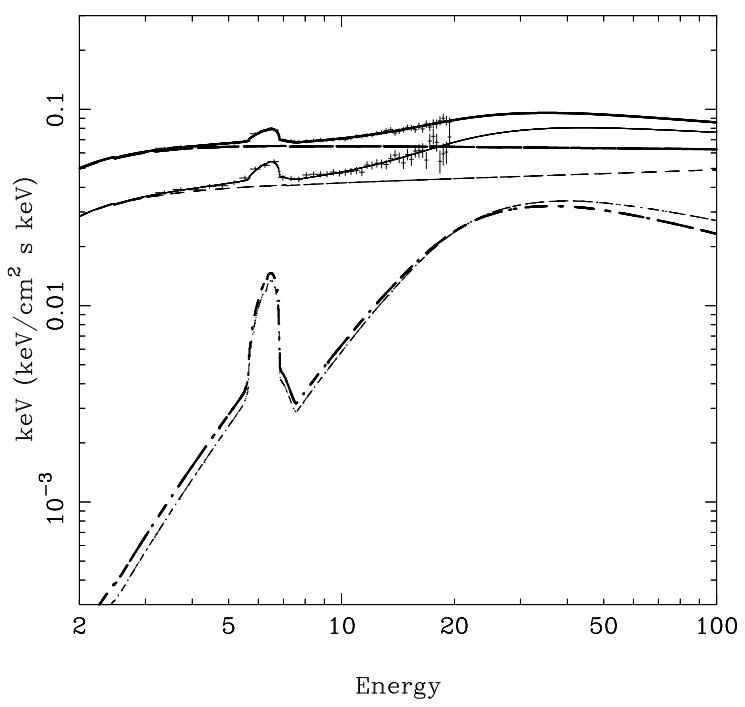

Fig. 6.- The high and low state PCA spectra fit to a simple power law and its relativistic reflection with absorption fixed at $3 \times 10^{21} \mathrm{~cm}^{-2}$. The best fit model components are shown in thick and thin lines for the high and low state spectra, respectively, where the spectral index and relative amount of reflection are $\alpha=2.00 \pm 0.02,1.92 \pm 0.04$, and $\Omega / 2 \pi=0.74 \pm 0.13,1.07_{-0.22}^{+0.25}$. The model is extrapolated out to $100 \mathrm{keV}$, showing that the high state power law has higher intensity at all energies of importance for the formation of the reflected continuum, despite being steeper (the pivot energy is at $\sim 1 \mathrm{MeV}$ ). 
angle of the relativistic reflector. The diagonal lines denote solutions where the power law index and reflected fraction remain constant between the two datasets. The power law index is clearly variable between the high and low flux level datasets irrespective of how the reprocessor is modelled. With a single reprocessor then the relative amount of reflection is only consistent with remaining constant at the $<90$ per cent confidence level. The data prefer a larger contribution of reflected flux relative to the power law in the low state spectrum i.e. that the absolute normalization of the reflected flux is constant. The dashed and dotted lines show the same contours for a model including an unsmeared, neutral reprocessed spectrum and Gaussian line from the molecular torus, respectively. The reflected fraction is then consistent with a constant value.

\subsection{PCA, HEXTE and OSSE Total Spectrum}

All the RXTE PCA data were co-added to form a single spectrum (with 1 per cent systematic uncertainty added) and fit together with the HEXTE and OSSE data to give a broad band spectrum. Current consensus is that the continuum is formed by Compton scattering of soft seed photons by hot electrons. Such a Comptonised continuum can be approximated by a power law with exponential cutoff, but this becomes inaccurate if the spectrum extends close to the energies of either the seed photons or hot electrons. The seed photons are presumably from the accretion disk, with expected temperatures of $\sim 10 \mathrm{eV}$, so the spectral curvature here is not an issue. However, the inclusion of the OSSE data means that the shape of the spectral cutoff from the electron temperature becomes important. Thus we use an analytical approximation to a Comptonised spectrum based on solutions of the Kompaneets equation (Lightman \& Zdziarski 1987), where the shape of the high energy cutoff is sharper than an exponential rollover.

The relativistic reflection and line from this incident continuum are calculated, and included in the model fit. The results are detailed in Table 3 for inclinations of the relativistic reprocessor of 30,60 and $72^{\circ}$ and iron abundances of 1 and $2 \times$ solar. We see the same trend as in Table 1 in terms of the spectra preferring higher iron abundance, and these solutions constrain the electron temperature to be $k T_{e} \sim 40-100 \mathrm{keV}$ (corresponding to an exponential e-folding energy of $\sim 120-300 \mathrm{keV})$. Figure 8 shows the best fit solution for twice solar abundance, inclined at $60^{\circ}$.

However, the poor signal-to-noise of the data at the highest energies means that this temperature is dependent on details of the reflection spectrum. For solar abundances the temperature is generally unconstrained.

We note that these data are consistent with previous observations of this source (Madejski et al. 1995), and with the mean Seyfert spectrum at high energies (Zdziarski et al. 1995). However, the temperatures of the Comptonizing medium derived from these data by Zdziarski et al. 1994) are rather higher $(k T \sim 250 \mathrm{keV})$, due to their assumption of an exponential rollover as an approximation to a Comptonised cutoff. This also means that the derived plasma optical depths in these previous papers of $\tau \sim 0.1$ are too low. Modelling the spectrum with more accurate 

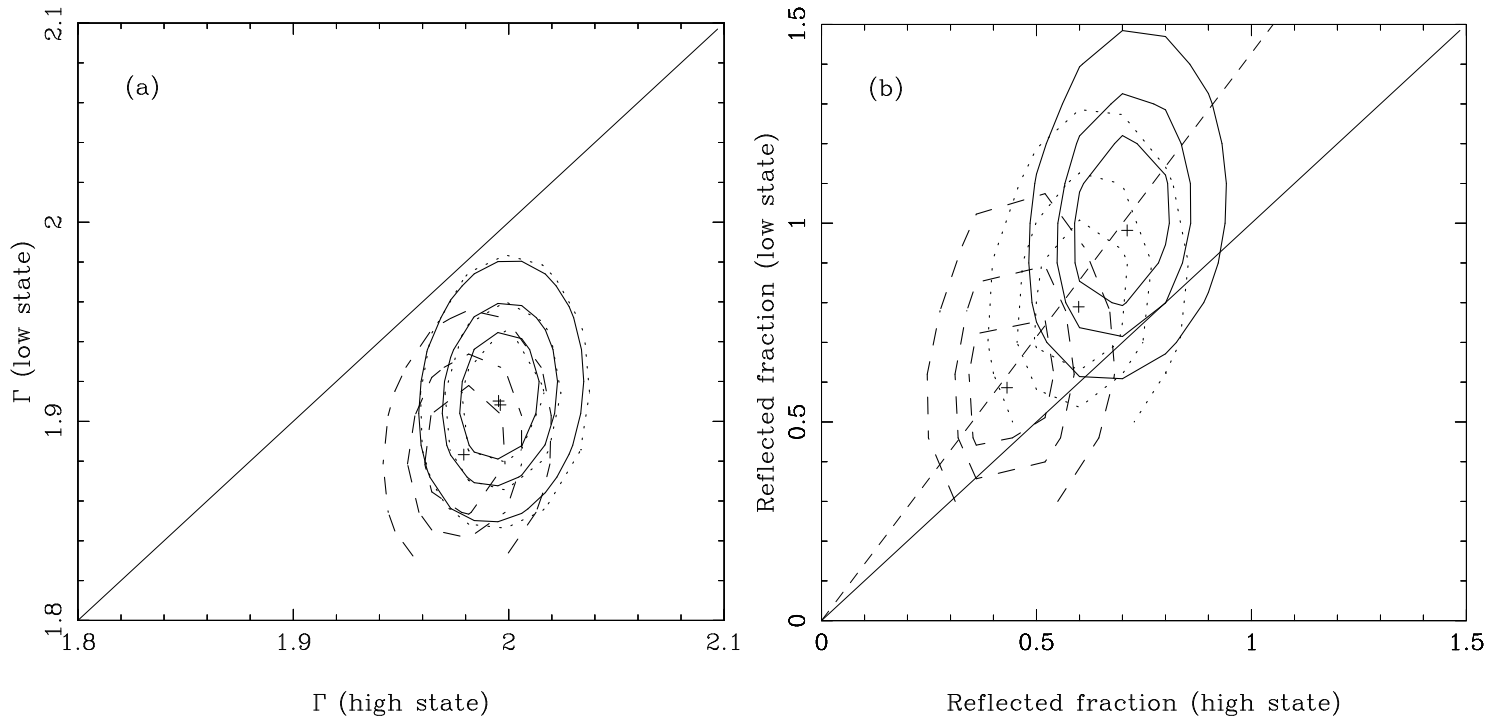

Fig. 7.- Confidence contours for (a) the power law spectral index and (b) the reflected fraction in the high and low state spectra. Solid contours denote a spectral model where the reprocessing is from an accretion disk, while the dashed contours include a fixed contribution from reflection from a molecular torus and the dotted contours include a fixed Gaussian line. In (a) the solid straight line denotes constant spectral index. This is plainly outside all the model contours, strongly requiring that the intrinsic power law index changes. In (b) the solid straight line indicates a constant reflected fraction, while the dashed line shows a constant absolute normalisation of the reflected component. Modeling the accretion disk alone gives confidence contours which are only marginally consistent at the 90 per cent confidence level with a constant reflected fraction. Instead the data prefer a constant absolute normalisation of the reflected component. Including a constant component (line or full reprocessed spectrum) shifts the derived contours for the accretion disk, allowing a constant reflected fraction at higher confidence level. 


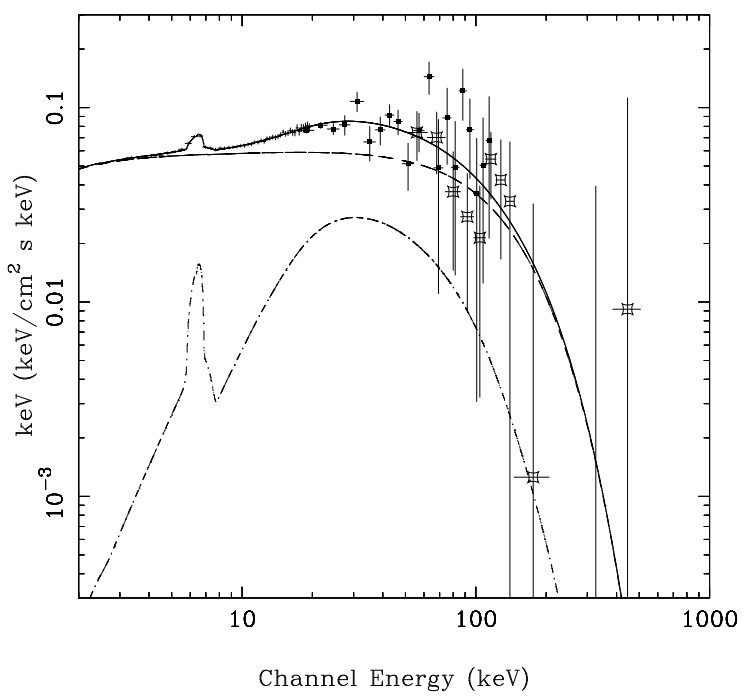

Fig. 8.- The total PCA (crosses), HEXTE (filled squares) and OSSE (open squares) spectra, fit to a comptonised continuum model with $k T_{e} \sim 50 \mathrm{keV}$, together with its relativistic reflection component for an assumed inclination of $60^{\circ}$ and twice solar iron abundance

Table 3. Total XTE PCA, RXTE HEXTE and CGRO OSSE data fit to an approximate comptonised continuum and its relativistically smeared reflection

\begin{tabular}{|c|c|c|c|c|c|c|c|c|}
\hline $\mathrm{A}_{\mathrm{Fe}}{ }^{\mathrm{a}}$ & Inclination & $\Gamma^{\mathrm{b}}$ & $k T_{e}(\mathrm{keV})$ & Norm at $1 \mathrm{keV}$ & $\Omega / 2 \pi^{\mathrm{c}}$ & $\xi^{\mathrm{d}}$ & $R_{\text {in }}{ }^{\mathrm{e}}\left(R_{\mathrm{g}}\right)$ & $\chi_{\nu}^{2}$ \\
\hline 1 & 30 & $1.97 \pm 0.03$ & $90_{-40}^{+360}$ & 0.053 & $0.48 \pm 0.10$ & $45_{-30}^{+45}$ & $23_{-14}^{+50}$ & $62.8 / 91$ \\
\hline 1 & 60 & $2.00 \pm 0.03$ & $110_{-57}^{+\infty}$ & 0.055 & $0.77_{-0.16}^{+0.17}$ & $13_{-8}^{+17}$ & $150_{-100}^{+\infty}$ & $68.2 / 91$ \\
\hline 1 & 72 & $2.01 \pm 0.03$ & $140_{-80}^{+\infty}$ & 0.056 & $1.20_{-0.25}^{+0.33}$ & $8_{-6}^{+11}$ & $160_{-100}^{+\infty}$ & $68.4 / 91$ \\
\hline 2 & 30 & $1.94 \pm 0.02$ & $50_{-15}^{+40}$ & 0.052 & $0.48_{-0.09}^{+0.10}$ & $10_{-9}^{+25}$ & $25_{-14}^{+75}$ & $59.6 / 91$ \\
\hline 2 & 60 & $1.96 \pm 0.03$ & $52_{-17}^{+46}$ & 0.053 & $0.75 \pm 0.16$ & $0.7_{-0.7}^{+8}$ & $110_{-55}^{+\infty}$ & $61.8 / 91$ \\
\hline 2 & 72 & $1.97 \pm 0.02$ & $55_{-20}^{+60}$ & 0.054 & $1.14_{-0.23}^{+0.21}$ & $0.01_{-0.01}^{+3}$ & $100_{-55}^{+\infty}$ & $60.3 / 91$ \\
\hline
\end{tabular}

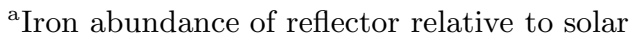

${ }^{\mathrm{b}}$ Photon spectral index

${ }^{\mathrm{c}}$ Solid angle subtended by the reprocessor with respect to the $\mathrm{X}$-ray source

${ }^{\mathrm{d}}$ Ionization parameter of the reprocessor

e Inner radius of the accretion disk 
comptonised spectra gives $k T \sim 130 \mathrm{keV}$ and $\tau \sim 1$ (Zdziarski et al. 1996).

\section{DISCUSSION}

\subsection{Fe K Line and Overall Spectral Shape}

Firstly, we clearly see that not all AGN are consistent with a substantial solid angle of extreme, relativistically smeared reflection. The reprocessed component seen in MGC-6-30-15 is not necessarily typical of AGN in general. A similar result is seen in a recent analysis of the ASCA spectrum of NGC 5548 (Chiang et al. 1999), where the line is broad, but not so broad as expected from a disk which extends down to the last stable orbit around a black hole (for the June 15 th data set they obtain $R_{\text {in }}=18.7_{-9.5}^{+29.1} R_{\mathrm{g}}$ for emissivity $\propto r^{-3}$; J. Chiang, private communication). Crucially, our data allow us to constrain a reflected component from a molecular torus. A torus with column $\geq 10^{23} \mathrm{~cm}^{-2}$ can produce a strong, narrow $6.4 \mathrm{keV}$ line, accompanied by a reflected continuum for columns $\geq 10^{24} \mathrm{~cm}^{-2}$. We do significantly detect such a contribution to the iron $\mathrm{K} \alpha$ line, which may also be accompanied by a reflected continuum. However, even with a narrow line from the torus, the remaining line from the accretion is not as broad as that seen in MCG-6-30-15.

The amount of relativistically smeared reflection is rather less than unity for any inclination $\leq 60^{\circ}$. Larger inclinations are not expected since this object is classified as a Seyfert 1 and no strong absorption from the torus is seen in the $\mathrm{X}$-ray spectrum. Thus the reflection from the accretion disk in IC4329a looks very like that seen in the low state spectra of the galactic black

hole systems (Życki et al. 1998; Done \& Życki 1999) in having $\Omega / 2 \pi<1$, relativistically smeared by velocities which are inconsistent with the reflecting material extending down to 3 Schwarzschild radii. There is then no intrinsic difference between the Galactic and extragalactic accreting black holes, but there is a spread in source properties in both classes (intrinsic spectral index, amount of reflection and amount of relativistic smearing). The AGN results to date show that steep spectra have a larger amount of reflection (Zdziarski et al. 1999) and more relativistic smearing. This is exactly the sequence seen in the Galactic Black Hole Candidate Nova Muscae 1991 (Życki et al. 1998; Życki et al. 1999) as a function of decreasing mass accretion rate.

There are currently two ways to explain the lack of extreme relativistic line. The first is to say that the inner accretion disk is simply not present, that it has been replaced by an X-ray hot flow. These composite truncated disk/hot X-ray source models were first proposed by Shapiro, Lightman and Eardley (1976) when they discovered a hot, two temperature, optically thin solution to the accretion flow equations, though this was subsequently shown to be unstable. Such models were given new impetus by the rediscovery of a related stable solution of the accretion flow equations (Narayan \& Yi 1995), which include advective as well as radiative cooling (ADAFs). These ideas are clearly consistent with our results. 
The alternative is that the inner disk is present, as required by the magnetic reconnection models for the X-ray flux, but that it cannot be seen in the reflected spectrum. One way to do this is if the upper layers of the disk are so ionized that they produce almost no atomic spectral features (Ross et al. 1999). Simple models for this, where the ionisation state of the disk varies as a smooth function of radius, do not match the data. However, the ionisation structure could be highly complex, with rapid transition between complete ionisation and relatively cool material (Różańska 1999; S. Nayakshin, private communication). Alternatively, if the X-ray source is moving away from the disk at transrelativistic velocities, perhaps because of plasma ejection from expanding magnetic loops, then its radiation pattern does not strongly illuminate the inner disk (Beloborodov 1999).

It is currently very difficult to distinguish observationally between these models, and all have some remaining theoretical problems. For the ADAF solutions, it is not yet known whether the fundamental assumptions underlying the solutions can hold, or how a transition from the cool disk to a hot flow can occur, while for the disk-corona geometry the uncertainties are mainly in the detailed outcome of magnetic reconnection, and in the ionisation structure of the illuminated disk.

\subsection{Spectral Variability}

Our data sample the source variability, which gives another way to investigate the underlying radiation mechanisms. This is only the second AGN where the reflected and intrinsic spectrum can be disentangled (the other is NGC 5548: Magdziarz et al. 1998; Chiang et al. 1999). The results show that the power law itself clearly gets intrinsically steeper as the source brightens, which allows to constrain the variability process. If there were merely more dissipation in the $\mathrm{X}$-ray hot corona without an accompanying change in soft seed photon flux then the spectrum would harden as it got brighter (e.g. Ghisellini \& Haardt 1994). Thus the observed spectral index-flux correlation implies that the soft seed photons also increase, and by somewhat more than the increase in the hard flux. Seed photons are thought to arise primarily through reprocessing, since the hard X-rays illuminating the disk which are not reflected are thermalised, emerging as soft photons. In this model the change in soft photons is commensurate with the change in flux dissipated in the hot corona. To change the seed photons by more than the change in hard dissipation requires either a change in geometry, such that the hard X-ray source intercepts a larger fraction of the disk radiation, or a decrease in reflection albedo, so that more of the incident hard X-ray radiation is thermalised rather than reflected. The former can be linked to the composite hot flow/cool accretion disk models as a result of varying the inner disk edge, while the latter could be produced in the disk-corona models if the ionisation state of the disk decreases for steeper spectra, so that the reflection albedo decreases and the thermalised soft flux increases. However, recent simultaneous observations of EUV and X-ray variability cast doubt on the simple scenario where the EUV seed photon flux is primarily reprocessed (Nandra et al. 1998; Chiang et

al. 1999). The variability that we see could equally well be the result of a variable soft photon flux 
irradiating the $\mathrm{X}$-ray region.

These three possibilities predict some differences in the behavior of the reflected continuum. If the disk geometry is changing to give more soft seed photons then we expect more solid angle of reflection as the spectrum steepens (as seen in the AGN/XRB compilation of Zdziarski et al. 1999). If the increased soft photons are from increasing thermalisation in the disk due to decreasing ionisation, then we should also see more cold reflection (as opposed to unobservable, completely ionized reflection) for steeper spectra. If it is simply the irradiating soft flux which is changing, without changing disk geometry then the reflected fraction should remain constant.

What we see is marginally (90 per cent confidence contour) consistent with a constant reflected fraction, although the data prefer that the relative amount of reflection decreases as the source increases and steepens. The resulting spectrum is consistent with the absolute normalisation of the reflected spectrum remaining constant as the source changes. Some part of the reflected spectrum could be contaminated by a line or reprocessed component from a molecular torus, which would be constant due to light travel time delays on the timescales of the monitoring campaign. Allowing for this results in the reflected fraction from the accretion disk being more convincingly consistent with a constant value, but still does not permit much of an increase with increasing flux or spectral index. The data then support the idea of a variable soft flux which is not reprocessed as the driver for the hard X-ray variability, but could also allow small changes in reflection geometry/ionisation.

We speculate that both variability mechanisms operate in Seyferts i.e. that there are spectral changes linked to changes in the geometry/ionisation (such as seen by Zdziarski et al. 1999), but that the soft seed photons can also vary independently of these changes, giving a second, subtly different source of spectral variability.

\section{CONCLUSIONS}

- Not all AGN have the extreme relativistic line profiles expected from a disk extending down to the innermost stable orbit around a black hole. This is consistent with either the inner disk being truncated before the last stable orbit, or with an inner disk which produces no significant reflected features either through anisotropic illumination or extreme ionisation. Simple photo-ionisation models, where the ionisation varies smoothly as a function of radius can be ruled out by the data, but these may differ substantially from more detailed models of the ionisation structure.

- There is intrinsic spectral variability, where the power law softens as the source brightens. This implies that the soft seed photons are increasing faster than the increase of the hard X-ray luminosity. The lack of a corresponding increase in the observed reflected spectrum implies that either the changes in disk inner radial extent/ionisation structure are small, or that the variability is actually driven by changes in the seed photons which are decoupled from the hard $\mathrm{X}$-ray 
mechanism.

\section{ACKNOWLEDGEMENTS}

This research was supported in part by grant 2P03D01816 of the Polish State Committee for Scientific Research (KBN) and by NASA grant NAG 54106. We thank James Chiang for

discussing with us their results on NGC 5548. We acknowledge the help of the OSSE team in reducing the OSSE data, and the RXTE Guest Observer Facility (and in particular, Tess Jaffe) for providing the PCA data reduction script rex.

\section{REFERENCES}

Beloborodov A.M. 1999, ApJ, 510, L123

Cappi M., Mihara T., Matsuoka M., Hayashida K., Weaver K.A., Otani C. 1996, ApJ, 458, 149

Chiang J., Reynolds C.S., Blaes O.M., Nowak M.A., Murray N., Madejski G.M., Marshall H.L., Magdziarz P. 1999, ApJ, in press

di Matteo T. 1998, MNRAS, 299, 15

Done C., Życki P.T. 1999, MNRAS, 305, 457

Esin A. A., McClintock J.E., Narayan R. 1997, ApJ, 489, 865

Fabian A.C., Rees M.J., Stella L., White, N.E. 1989, MNRAS, 238, 729

Galeev A.A., Rosner R., Vaiana G.S. 1979, ApJ, 229, 318

George I.M., Fabian A.C. 1991, MNRAS, 249, 352

George I.M., Turner T.J., Netzer H., Nandra K., Mushotzky R.F., Yaqoob T. ApJS, 114, 73

Ghisellini G., Haardt F. 1994, ApJ, 429 L53

Ghisellini G., Haardt F., Matt G. 1994, MNRAS, 267, 743

Gierliński M., Zdziarski A. A., Done C., Johnson W. N., Ebisawa K., Ueda Y., Phlips F. 1997, MNRAS, 288, 958

Gierliński M., Zdziarski A. A., Poutanen J., Coppi P. S., Ebisawa K., Johnson W. N. 1999, MNRAS, in press

Haardt F., Maraschi L., Ghisellini G. 1994, ApJ, 432, 95

Henry R.B.C., Worthey G. 1999, PASP, 111, 919 
Iwasawa K., Fabian A.C., Mushotzky R.F., Brandt W.N., Awaki H., Kunieda H. 1996, MNRAS, 279,837

Janiuk A., Życki P.T., Czerny B. 1999, MNRAS, submitted

Johnson W.N., Zdziarski A.A., Madejski G.M., Paciesas W.S., Steinle H., Lin Y.-C., 1997, in Dermer C. D., Strickman M. S., Kurfess J. D., eds, The 4th Compton Symposium, AIP, New York, 283

Krolik J.H., Madau P., Życki P.T. 1994, ApJ, 420, L57

Lightman A.P., White T.R. 1988, ApJ, 335, 57

Lightman A.P., Zdziarski A.A. 1987, ApJ, 319, 643

Madejski, G.M., Sikora, M., Jaffe, T., Błażejowski, M., Jahoda, K., Moderski, R. 1999, ApJ, in press

Madejski, G. M., et al. 1995, ApJ, 438, 672

Magdziarz P., Zdziarski A.A., 1995, MNRAS, 273, 837

Magdziarz P., Blaes O.M., Zdziarski A.A., Johnson W.N., Smith D.A. 1998, MNRAS, 301, 179

Makishima K., et al., 1996, PASJ, 48, 171

Matt G., Perola G.C., Piro L. 1991, A\&A, 247, 25

Matt G., Fabian A.C., Ross R., 1993, MNRAS, 262, 179

Morrison R., McCammon D. 1983, ApJ, 270, 119

Mushotzky R.F., Fabian A.C., Iwasawa K., Kunieda H., Matsuoka M., Nandra K., Tanaka Y. 1995, MNRAS, 272, 9p

Nandra K., Pounds K.A. 1994, MNRAS, 268, 405

Nandra K., George I.M., Mushotzky R.F., Turner T.J., Yaqoob T. 1997, ApJ, 477, 602

Nandra K., Clavel J., Edelson R.A., George I.M., Malkan M.A., Mushotzky R.F., Peterson B.M., Turner T.J. 1998, ApJ, 505, 594

Narayan R., Yi I. 1995, ApJ, 444, 231

Pounds K.A., Nandra K., Stewart G.C., George I.M., Fabian A.C. 1990, Nature, 344, 132

Press W.H., Teukolsky S.A., Vettering W.T., Flannery B.P. 1992, Numerical Recipes (New York: Cambridge University Press)

Reynolds C.S. 1997, MNRAS 286, 513

Ross R.R., Fabian A.C., Young A.J. 1999, MNRAS, 306, 461 
Rothschild, R.E., et al. 1998, ApJ, 496, 538

Różańska A. 1999, MNRAS, 308, 751

Rybicki G.B., Lightman A.P. 1979, Radiative Processes in Astrophysics (New York: WileyInterscience)

Shakura, N.I., Sunyaev, R.A. 1973, A\& A, 24, 337

Shapiro S.L., Lightman A.P., Eardley D.M. 1976, ApJ, 204, 187

Tanaka Y. et al. 1995, Nature, 375, 659

Toor A., Seward F.D., 1974, AJ., 79, 995

Weaver K.A., Yaqoob T., Mushotzky R.F., Nousek J., Hayashi I., Koyama K. 1997, ApJ, 474, 675

Weaver K.A., Reynolds C. 1998, ApJ, 508, L39

Zdziarski A.A., Fabian A.C., Nandra K., Celotti A., Rees M.J., Done C., Coppi P., Madejski G.M. 1994, MNRAS, 269, 55p

Zdziarski A.A., Johnson W.N., Done C., Smith D.A., McNaron-Brown K. 1995, ApJ, 438, 63

Zdziarski A.A., Gierliński M., Gondek D., Magdziarz P. 1996, A\&AS, 120, 553

Zdziarski A.A., Lubiński P., Smith D.A. 1999, MNRAS, 303, L11

Zhang, W., Giles, A.B., Jahoda, K., Swank, J. H, Morgan, E.M. 1993, in "EUV, X-ray, and

Gamma-ray Instrumentation for Astronomy IV," SPIE Proceedings, O. Siegmund ed., 2006, 324

Życki P.T., Done C., Smith D.A. 1997, ApJ, 488, L113

Życki P.T., Done C., Smith D.A. 1998, ApJ, 496, L25

Życki P.T., Done C., Smith D.A. 1999, MNRAS, 305, 231 